\title{
Natural killer cells and pigment epithelial- derived factor control the infiltrative and nodular growth of hepatic metastases in an Orthotopic murine model of ocular melanoma
}

\author{
Nyasia M. Jones ${ }^{1}$, Hua Yang ${ }^{2}$, Qing Zhang ${ }^{2}$, Vanessa M. Morales-Tirado ${ }^{3,4+}$ and Hans E. Grossniklaus ${ }^{2,5^{*}}$
}

\begin{abstract}
Background: Metastases account for $90 \%$ of all cancer-related deaths, becoming a therapeutic problem. Approximately $50 \%$ of all uveal melanoma (UM) patients will develop metastases, mainly in the liver. Post-mortem analyses of livers from metastatic UM patients showed two different metastatic growth patterns: infiltrative and nodular. The infiltrative pattern exhibits tumor infiltration directly to the hepatic lobule and minimal angiogenesis. The nodular pattern shows clusters of tumor cells around the portal venules that efface the liver parenchyma. We recently demonstrated Natural Killer (NK) cells play a pivotal role in the control of hepatic metastases and the pigment epithelial-derived factor (PEDF) controls angiogenesis in the liver using our established ocular melanoma animal model. In this study we investigated the role of NK cells and PEDF in the development of metastatic growth patterns, as this can contribute to the development of novel therapeutics specific towards each growth pattern.

Methods: We utilize our established ocular melanoma animal model by inoculation of B16-LS9 melanoma cells into C57BL/6 J mice (WT), anti-asialo GM1-treated C57BL/6 J mice (NK-depleted), and PEDF ${ }^{-1-}$ C57BL/6 J mice. Three weeks after inoculation we evaluated the metastatic growth patterns and stratified them based of the numbers of tumor cells. To evaluate angiogenesis the mean vascular density (MVD) was calculated. The immune compartment of the liver was analyzed by flow cytometry.

Results: Our in vivo work showed two distinct metastatic growth patterns, the infiltrative and nodular, recapitulating the post-mortem analyses on human liver tissue. We discovered NK cells control the infiltrative growth. In contrast, PEDF controlled anti-angiogenic responses, showing higher MVD values compared to NKdepleted and WT animals. The myeloid lineage, comprised of monocytes, macrophages, and myeloid-derived suppressor cells, was reduced in the absence of NK cells or PEDF.
\end{abstract}

(Continued on next page)

\footnotetext{
*Correspondence: ophtheg@emory.edu

${ }^{\dagger}$ Vanessa M. Morales-Tirado and Hans E. Grossniklaus are Both authors are Senior Co-authors.

${ }^{2}$ Department of Ophthalmology, Emory University School of Medicine,

Atlanta, GA 30322, USA

${ }^{5}$ Winship Cancer Institute at Emory University, 1365 Clifton Road NE, BT428,

Atlanta, GA 30322, USA

Full list of author information is available at the end of the article
}

(c) The Author(s). 2019 Open Access This article is distributed under the terms of the Creative Commons Attribution 4.0 International License (http://creativecommons.org/licenses/by/4.0/), which permits unrestricted use, distribution, and reproduction in any medium, provided you give appropriate credit to the original author(s) and the source, provide a link to the Creative Commons license, and indicate if changes were made. The Creative Commons Public Domain Dedication waiver (http://creativecommons.org/publicdomain/zero/1.0/) applies to the data made available in this article, unless otherwise stated. 
(Continued from previous page)

Conclusions: Our animal model recapitulates the metastatic growth patterns observed in the human disease. We demonstrated a role for NK cells in the development of the infiltrative growth pattern, and a role for PEDF in the development of the nodular pattern. The understanding of the complexity associated with the metastatic progression has profound clinical implications in the diagnostic and disease-management as we can develop and direct more effective therapies.

Keywords: Hepatic metastases, Ocular melanoma, Tumor dissemination, NK cells, PEDF, Animal models

\section{Background}

Uveal Melanoma (UM) is the most common intraocular malignancy in adults $[1,2]$. Despite effective control of the primary tumor, about $50 \%$ of UM patients develop metastatic disease. The mortality rate of these patients has not significantly changed in the last four decades due to the lack of an effective clinical treatment against metastatic disease [2]. The liver is the main site of primary metastasis in over $75 \%$ of cases, as UM tumor cells disseminate hematogenously $[1,3]$, and the CXCR4 [46] and c-Met $[7,8]$ receptors, which are present in UM, mediate migration toward ligand gradients produced by the liver. Studies aiming to understand the mechanisms and patterns of UM dissemination within the liver are limited. We recently identified two types of metastatic growth pattern through post-mortem analyses in human livers of metastatic UM patients: nodular and infiltrative [9]. The nodular pattern is characterized for growing adjacent to the portal venule effacing the surrounding hepatic parenchyma. The hepatocytes are pushed aside destroying the pre-existing liver architecture. These hepatocytes are separated from the tumor cells by a thin layer of reticulin fibers. In contrast, the infiltrative pattern shows invasion of the hepatic lobule, replacing healthy hepatocytes [9]. The metastatic cells invade the liver parenchyma without disturbing the pre-existing liver structure at the interface [10].

The landscape of metastatic progression is of great importance for the understanding of the interplay between tumor cells and the microenvironment. Niederkorn and colleagues [11] initially reported NK cell activity in the eye promoted the growth of UM. A follow up in vitro study by these authors suggested macrophage migration inhibitory factor (MIF) production by UM cells protects against NK cell-mediated killing [12]. Our group developed an ocular melanoma murine model and we formally demonstrated NK cells are pivotal for the control of hepatic metastases [13].

As the role of PEDF in suppression of ocular neovascularization was elucidated, our group discovered that the ratio of vascular endothelial growth factor (VEGF) to PEDF played a role in the migration of UM cells and hepatic metastases [14]. Furthermore, we recently demonstrated the role of PEDF as an anti-angiogenic and anti-stromagenic factor in UM [15]. Still, the role on NK cells and PEDF in the development of metastatic growth patterns and immune polarization is not understood. To evaluate the roles of NK cells and PEDF in the tumor microenvironment in metastatic UM in the liver, we compared the tumor microenvironment relative to metastatic UM growth using our established orthotopic murine model of ocular melanoma. Our results suggest a role for NK cells in the development of the infiltrative metastatic growth pattern and a role for PEDF in the nodular growth. We measured a reduction in the myeloid lineage within the metastatic liver and discovered the expression of both pro-inflammatory and anti-inflammatory genes.

\section{Methods}

\section{Tumor and cell culture conditions}

The mouse melanoma cell line B16-LS9 was kindly provided by Dario Rusciano at the Friedrich Miescher Institut, Basel, Switzerland. The complete culture medium included RPMI1640 with HEPES, L-glutamine, 10\% FBS, 1\% nonessential amino acids, $1 \%$ sodium pyruvate solution, $1 \%$ MEM vitamin solution, and a $1 \%$ antibiotic-antimycotic solution and incubated at $37{ }^{\circ} \mathrm{C} / 5 \% \mathrm{CO}_{2}$. Cells were grown to $90 \%$ confluence prior to harvest, washed with Hank's solution, trypsinized and expanded for experiment use.

\section{Mice}

Eight-week old female C57BL/6 J mice were purchased from Jackson Laboratories (Bar Harbour, ME). $P E D F^{-1-}$ mice were generated on a C57BL/6 background as previously described [16] and provided courtesy of Dr. Susan Crawford (St. Louis University, St. Louis, MO). All experiments were performed according to the Declaration of Helsinki, in compliance with the Association for Research in Vision and Ophthalmology (ARVO) Statement for the use of animals in Ophthalmic and Visual Research and with the Institutional Animal Care and Use Committee policies and procedures from Emory University.

\section{Anti-Asialo GM1-mediated NK cell depletion}

A cohort of 15 female C57BL/6 J mice was treated with anti-asialo GM1 serum to deplete NK cells. Anti-asialo GM1 (Rabbit; Wako Pure Chemical Industries, Osaka, Japan) was diluted to $1: 4$ in distilled water prior to 
injection. To achieve NK cell depletion mice were injected intraperitoneally (i.p.) with $100 \mu \mathrm{L}$ anti-asialo GM1 every 3 days, beginning two weeks prior to cell line inoculation until they were euthanized [17].

\section{Murine model of ocular melanoma}

Eight-week old female C57BL/6 J, C57BL/6 PEDF ${ }^{-1}$, and C57BL/6 J anti-asialo GM1-treated mice were inoculated in the posterior compartment of the right eye with $5 \times 10^{5}$ B16-LS9 melanoma cells in a $2.5 \mu \mathrm{L}$ final volume using a transcorneal technique with a 30-gauge needle under guidance of a dissection microscope. The mice were anesthetized by intraperitoneal injection (i.p.) of $100 \mathrm{mg} / \mathrm{kg}$ ketamine and $12 \mathrm{mg} / \mathrm{kg}$ xylazine mixture in PBS. The inoculated eyes were enucleated after one-week. Mice were euthanized in a $\mathrm{CO}_{2}$ chamber 28 days post inoculation. Liver tissue were collected and processed for histological and cellular evaluation.

\section{Histology assessment}

Excised livers were grossly examined and submerged in $4 \%$ neutral buffered formaldehyde for routine processing. Formalin fixed-paraffin embedded slides were stained with hematoxylin and eosin (H\&E) and microscopically evaluated (Olympus, Waltham, MA) for metastases. Three sections through the center of each liver were evaluated for the number of metastases, and the average number per section was determined, as previously described $[17,18]$. To determine relative metastasis area the sizes of all metastases in a single liver section were added to calculate a total metastasis area $\left(\mu \mathrm{m}^{2}\right)$ and averaging this value per mouse. Metastases were separated by size into stage $1(<50 \mu \mathrm{m}$ in diameter), stage 2 $(50-200 \mu \mathrm{m})$, and stage 3 macrometastases $(>200 \mu \mathrm{m})$. Measurements were performed in triplicate and averaged per mouse as previously described [19].

\section{Evaluation of tumor vascular density}

The number of blood vessels per 40x high-powered field (HPF) was calculated and averaged to mean vascular density (MVD). An individual vessel was defined as an area of lumen lined with endothelial cells, while tracts and branches were counted as separate vessels. C57BL/6 J, $n=$ 9; C57BL/6 $P E D F^{-/-}, \mathrm{n}=9$; and $\mathrm{C} 57 \mathrm{BL} / 6 \mathrm{~J}$ anti-asialo GM1-treated mice, $n=8$.

\section{Cell suspension from liver tissue}

Murine livers were isolated by maceration using a pestle (MidSci, St. Louis, MO) on a $70 \mu \mathrm{m}$ nylon strainer (BD Biosciences, San Jose, CA). Cell suspension was washed and red blood cell contaminants were eliminated using 1x RBC Lysis Buffer (BioLegend, San Diego, CA) following manufacturers instructions. Cells were resuspended in $\mathrm{PBS} / 1 \%$ FBS and kept on ice until ready to use.
Cell surface labeling for flow cytometry analyses

Cell suspension was labeled with the following antibodies: anti-CD11b (clone M1-70) PE, anti-Ly-6C (clone HK1.4) FITC, anti-Ly-6G (clone 18A) APC, anti-Gr-1 (clone RB6-8C5) FITC, and anti-F4/80 (clone BM8) PE. All anti-mouse antibodies were purchased from BioLegend. We labeled each sample with $1 \mu \mathrm{g}$ of each antibody and incubated on ice for $30 \mathrm{~min}$ in dark. Samples were washed $3 \mathrm{x}$ in PBS/1\% FBS. Acquisition strategy: $\mathrm{CD}_{11 \mathrm{~b}^{+} \text {cells }}$ (myeloid lineage): we gated FSC versus SSC to eliminate debris (Gate 1), followed by plotting of CD11b versus SSC

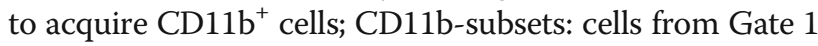
were plotted CD11b versus Ly6C, or Ly6G, or Gr-1; Kupffer cells: F4/80 versus SSC. Immunopositivity was determined by using isotype controls of each antibody and unlabeled samples. Single label controls were set up using The $\mathrm{AbC}^{\mathrm{m}}$ Total Antibody Compensation Bead Kit (Thermo Fisher Scientific, Waltham, MA). Data acquisition was done in BD Biosciences LSRII Flow Cytometer and data analyses performed using FlowJo vX.0.0.6 (FlowJo, LLC, Ashland, OR), as before [20].

\section{Gene expression analyses}

RNA was extracted from cells or tissue using RNeasy Mini Kit (Qiagen Inc., Valencia, CA) following manufacturer's conditions. We used $100 \mathrm{ng}$ of RNA material for cDNA synthesis and further pre-amplification prior to assay set up to increase test sensitivity. Samples were run in Roche ${ }^{\odot}$ LightCycler 480 and analyzed using the Comparative $\Delta C_{T}$ Method as before [21]. We used the following assays (Thermo Fisher Scientific): Tnfa: Mm00443258_m1; Socs3: Mm00545913_s1; Arg1: Mm00 475988_m1; Tgfb1: Mm01178820_m1; Gapdh: Mm 99999915_g1; Casp3: Mm01195085_m1; Trp53: Mm 01731290_g1; Cd68:Mm03047340_m1.

\section{Statistical analysis}

A one-way ANOVA or two-way ANOVA with Tukey's post-test was performed to determine statistical significance. Experiments comparing the immune cell populations used The Holm-Sidak test. The value $p<0.05$ was used to define statistical significance for all assays. All data was reported as mean \pm SEM using Prism Graph Pad (GraphPad Software, La Jolla, CA).

\section{Results}

Increased number of hepatic metastases in the absence of NK cells and PEDF production

We investigated the average number of hepatic metastases in our orthotopic model in the absence of NK cells and PEDF. The B16-LS9 melanoma cell line was inoculated into the posterior compartment of wild type C57BL/6 J mice (WT), C57BL/6 J mice treated with anti-asialo GM1 serum to deplete NK cells (NK-depleted) and $P E D F^{-/-}$ 
C57BL/6 J mice $\left(P E D F^{-/}\right)$. Hepatic metastases were counted after 3 -weeks post inoculation and compared in all groups. We measured a significant increase in the average number of metastases in the NK-depleted ${ }^{* * * *} p<$ $0.001)$ and $P E D F^{-1}\left({ }^{* * * * *} p<0.001\right)$ groups compared to WT controls (Fig. 1a). Next, we stratified the metastases based on a 3-staging system where stage 1 includes metastases $<50 \mu \mathrm{m}$ in size, stage 2 is between 50 and $200 \mu \mathrm{m}$, and stage 3 comprised $>200 \mu \mathrm{m}$ in size. As shown in Fig. $1 \mathrm{~b}$, WT livers showed a predominant stage 1 while NK depleted and $P E D F^{-1}$ groups had increased numbers of stage 2 and 3 metastases. A more detailed comparison between NK depleted and $P E D F^{-/}$groups revealed a predominance of stage 2 in the NK depleted group and higher numbers of both stage 2 and 3 compared to the $P E D F^{-1}$ group. Representative images of the stages of the hepatic metastases are shown in Fig. 1c. The tumors were grossly visible upon examination, as shown in Fig. 1d.

\section{Inhibitory effect of PEDF on the nodular pattern of metastatic growth}

We sought to investigate the contribution of NK cells or PEDF in UM metastatic growth patterns (Fig. 2a) by measurement of the ratio of infiltrative: nodular growth. WT animals measured an infiltrative: nodular ratio of 3:1, similar to our post-mortem analyses on human livers from metastatic UM patients [9]. This ratio changed in the absence of NK cells towards an equal proportion of infiltrative and nodular growth patterns. In the absence of PEDF there was an increased ratio of nodular to infiltrative growth pattern compared to both WT and NK depleted groups $\left({ }^{* * * *} p<0.001\right)$. Next, we stratified the growth patterns based on the staging system described in Fig. 1. We measured a significant increase in the average number of metastases following the infiltrative growth pattern in stage $2\left({ }^{*} p<0.05\right)$ and $3\left({ }^{* * *} p<0.005\right)$ in the NK depleted group compared to WT (Fig. 2b, left). The $P E D F^{-/}$group showed a significant increase in the average number of metastases following the infiltrative patter in stage $2(* p<0.005)$ compared to WT. There was a significant increase in the examination of nodular growth across all stages in both NK depleted (stage 1: ${ }^{*} p<0.05$; stage 2: *** $p<0.005$; stage $3:{ }^{*} p<0.05$ ) and $P E D F^{-1-}$ (stages $1-3$ : $* p<0.05$ ) groups compared to WT, as shown in Fig. 2b, right. WT control showed no stage 3 metastases, confirming our findings shown in Fig. 1b. Representative images of each stage within the infiltrative or the nodular growth patterns is shown in Fig. 2c. The infiltrative growth pattern showed metastatic cells within the hepatic lobule (Fig. 2c, upper panels). In contrast, the nodular growth pattern exhibited metastatic cells adjacent the portal venule (Fig. 2c, lower panels), represented by the white arrow.

Mean vascular density (MVD) was investigated as an angiogenesis measurement suggested correlates with metastatic development. In Fig. $2 \mathrm{~d}$ we compared the MVD of metastatic tissue by counting the number of blood vessels per 40x high power field of magnification (40HPF). Our results showed a significant increase in MVD in the NK depleted $\left({ }^{* * * * *} p<0.00005\right)$ group compared to WT. The PEDF ${ }^{-/}$group $(* * * *<<0.00005)$ showed a significant increase in MVD compared to NK depleted and to WT animals.

\section{NK cell depletion and PEDF inhibition are independently associated with a reduction in myeloid lineage}

The interplay between the tumor cells and the microenvironment is of great importance for the understanding of the metastatic progression. We investigated if the absence of NK cells or PEDF in addition to favor a metastatic growth pattern, it may exhibit a polarized type of immune response. A number of markers have been

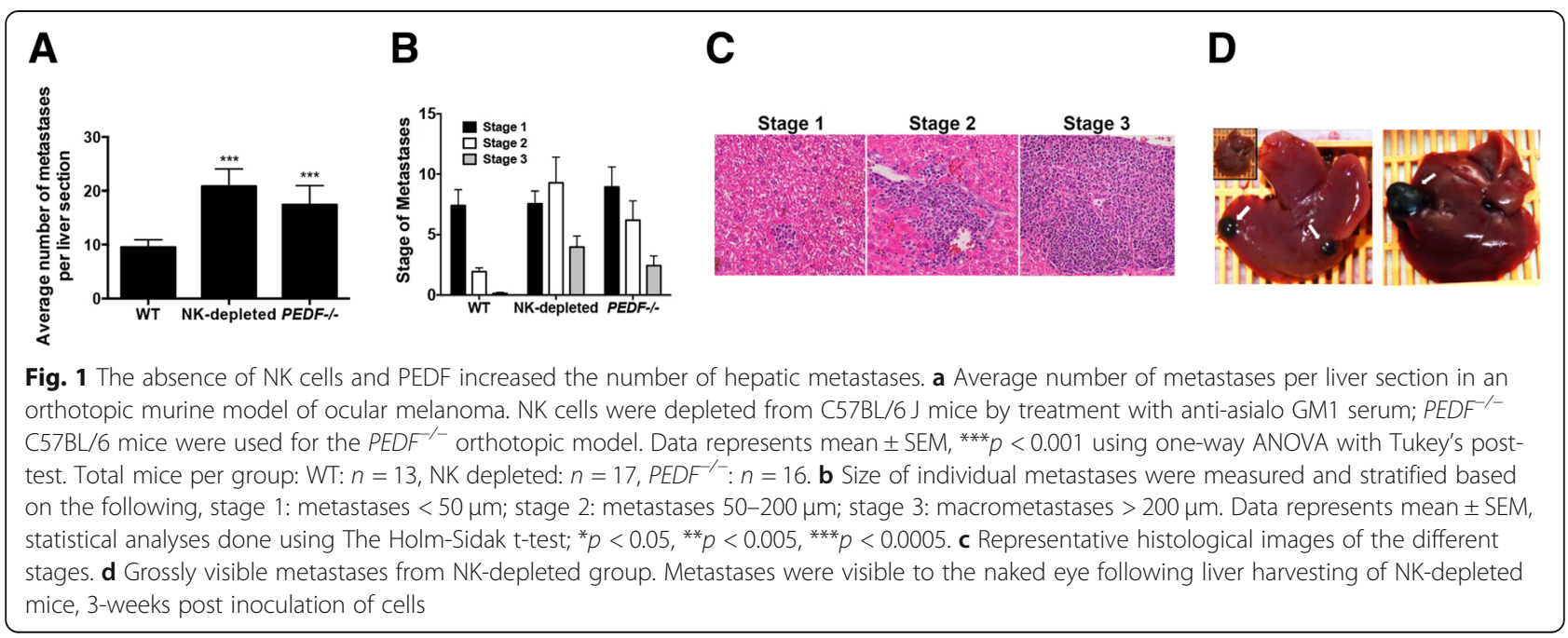




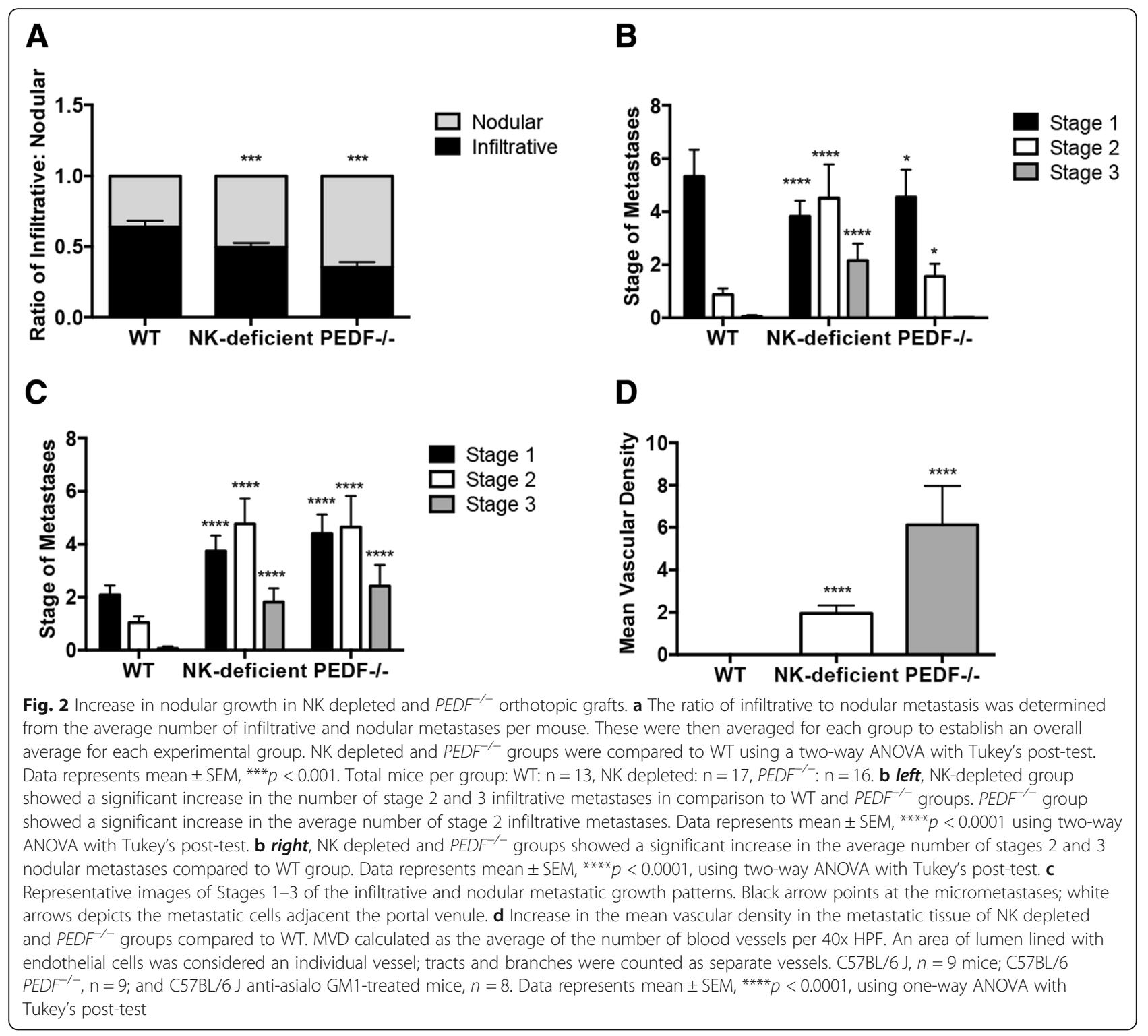

identified for the M1 or pro-inflammatory macrophages and the M2 involved in tissue remodeling and immune regulation. We measured Tnf and Socs3 mRNA expression by qPCR analyses to indicate M1 polarization in the liver tissue from all groups. Our results shown in Fig. 3a demonstrated higher expression of Tnf mRNA in WT compared to the other groups. There was $50 \%$ reduction in the NK depleted and $P E D F^{-/-}$groups compared to WT. Measurement of Socs3 mRNA revealed higher expression in WT and NK depleted groups compared to the $P E D F^{-1-}$ group. Next, we chose Arg1 and Tgfb as genetic markers for M2 polarization. All groups measured $\operatorname{Arg} 1$ mRNA, while only the WT and NK depleted groups measured $T g f b$ mRNA expression. The liver microenvironment showed mRNA expression of M1 and M2-type genes.
Next, we investigated the tumor suppressor mechanisms associated with the hepatic metastatic growth in the absence of NK cells and the absence of PEDF. Both Casp 3 and Trp53 genes were downregulated in the NK-depleted and $P E D F^{-1}$ animals compared to WT. Moreover, the reduction in these genes was stronger in the absence of PEDF, as shown in Fig. 3b.

Our interest in the role of different myeloid populations in the clearance of hepatic metastases led us to first, investigate the gene expression of $C D 68$, a biomarker of infiltrative monocytes, in the liver microenvironment. We measured high expression of CD68 mRNA across all groups. The expression was higher in the NK-depleted and $P E D F^{-/}$groups compared to WT (Fig. 3c). In Fig. 4a we measured the percentage of live $\mathrm{CD} 11 \mathrm{~b}^{+}$, which comprise monocytes, neutrophils, dendritic cells, and some 


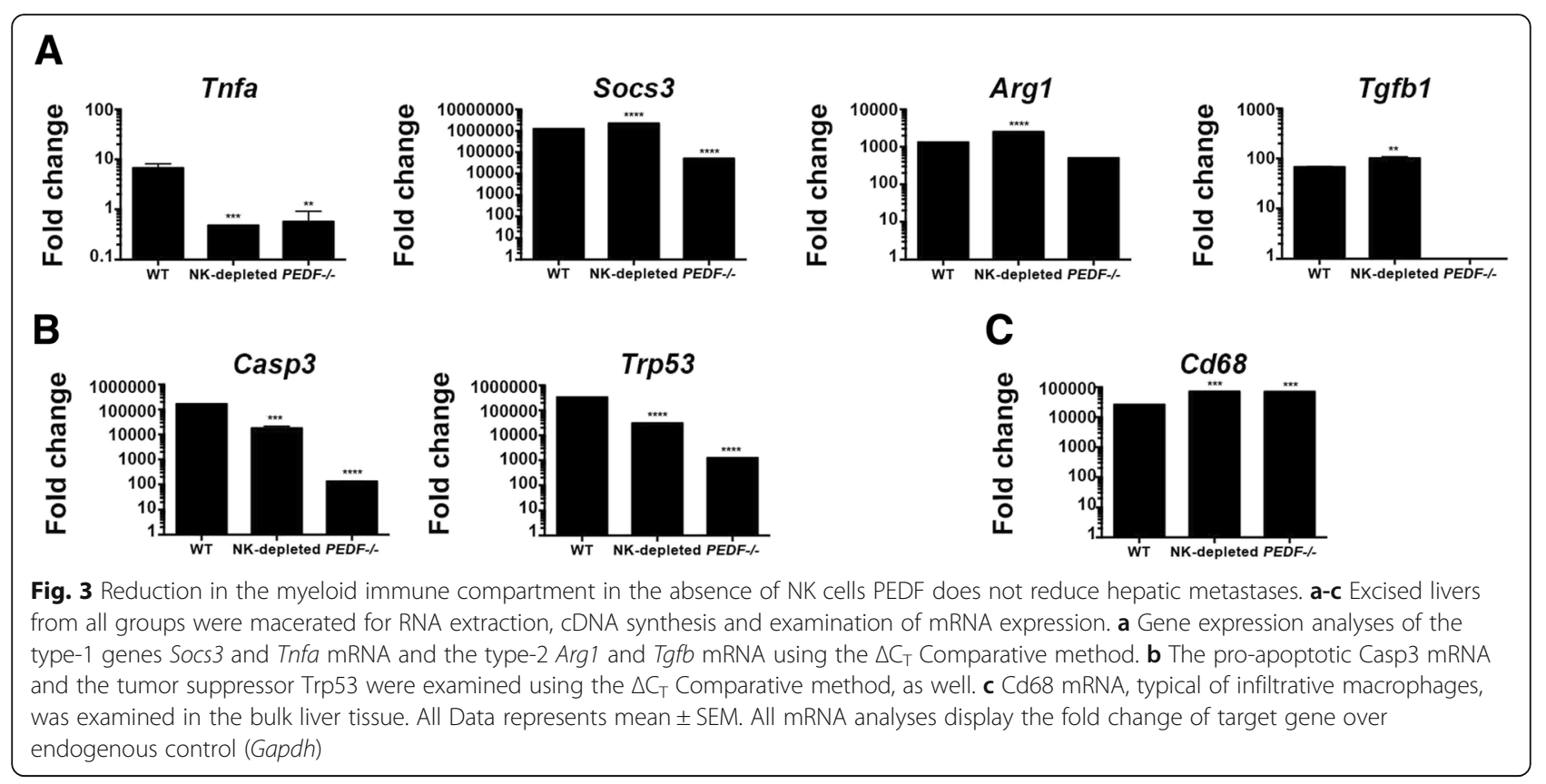

NK cells [22-25], to investigate if the increase in hepatic metastases and angiogenic activity was due to modulation of this population. Figure 4a, lower panel showed a small increase in $\mathrm{CD}_{11} \mathrm{~b}^{+}$cells in the NK-depleted livers. Representative pseudocolor plots of each group are shown in Fig. 4a, upper panel. Myeloid derived suppressor cells (MDSCs) have distinct phenotypes based on Ly6C or Ly6G expression. There was a striking reduction, albeit not significant, in the percentage of live $\mathrm{CD}_{11 \mathrm{~b}^{+} \mathrm{Ly} 6 \mathrm{C}^{+}}$ cells in the NK-depleted and $P E D F^{-/}$groups compared to WT (Fig. 4b). However, there was a significant reduction in the percentage of live $\mathrm{CD} 11 \mathrm{~b}^{+} \mathrm{Ly}_{6 \mathrm{G}^{+}}$cells, which are considered neutrophils, in the NK-depleted $\left({ }^{*} p<0.05\right)$ and $P E D F^{-1}(* p<0.05)$ groups compared to WT (Fig. 4c). Quantitation of cells with the immature myeloid

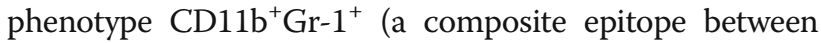
the Ly6C and Ly6G antigens) revealed a significant reduction in the percentage of live $\mathrm{CD} 11 \mathrm{~b}^{+} \mathrm{Gr}-1^{+}$cells in the NK depleted $(* p<0.05)$ and $P E D F^{-1-}\left({ }^{* * *} p<0.005\right)$ groups compared to WT (Fig. 4d). The percentage of live Kupffer cells with a phenotype of $\mathrm{F} 4 / 80^{+}$cells was significantly reduced in the NK depleted $(* * * * 0.0005)$ and $P E D F^{-/-}$ $(* *<0.005)$ groups compared to the WT (Fig. 4e). Collectively, our results showed depletion of NK cells or absence of PEDF reduces the percentages of MDSC and correlates with an increase in hepatic metastases.

\section{Discussion}

Our findings suggest NK cells and PEDF play a role in the growth pattern of metastatic UM. Hepatic metastases are more abundant in the absence of NK cells and PEDF. Moreover, we characterized for the first time the growth patterns of metastatic UM in an orthotopic murine model of ocular melanoma. The WT group shows a higher ratio of infiltrative to nodular metastatic pattern similar to that observed in post mortem human livers from metastatic UM patients [9]. The ratio of infiltrative to nodular is reduced in the absence of NK cells and PEDF. Divergence among these two was observed in the studies characterizing the microenvironment and in the tumor vascular supply. NK cells play a role in the microenvironment of the hepatic metastases while PEDF is more critical for the angiogenesis.

We have previously demonstrated that the hepatic sinusoidal space contains resident NK cells [9]. However, these NK cells are in an immature state. Recent investigations from our group showed the Toll-like receptor-5 (TLR-5) agonist entolimod enhanced anti-metastatic activity against hepatic metastases by mobilization of NK cells to the liver, and stimulate the maturation, differentiation and activation of the resident ones [13]. Because of the presence of NK cells in the sinusoidal space within the infiltrative pattern of metastatic growth we hypothesized that depletion of this population will shift the ratio of infiltrative to nodular, increasing the nodular pattern. Our results supported our hypothesis as it increased the number of metastases following the nodular pattern. This increase in nodular pattern shifted the ratio to a 1:1, showing a similar distribution of infiltrative and nodular patterns. The staging or stratification of the hepatic metastases also differed in the absence of NK cells. In both the infiltrative and nodular patterns the hepatic metastases followed the patter of higher stage $2>$ stage $1>$ stage 3 , compared to the WT group, which 
A
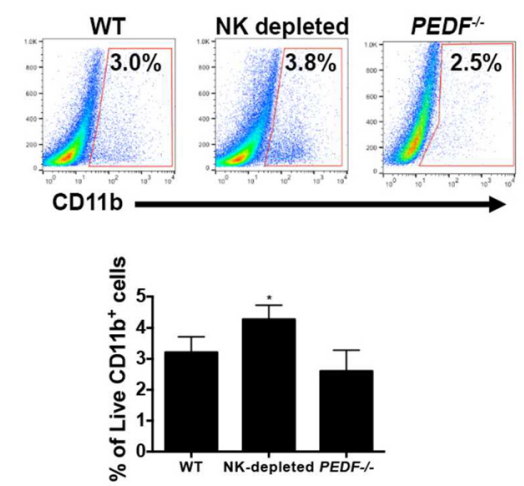

C

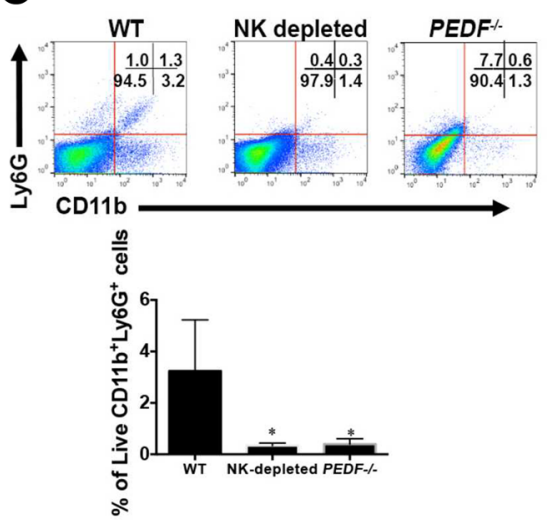

E

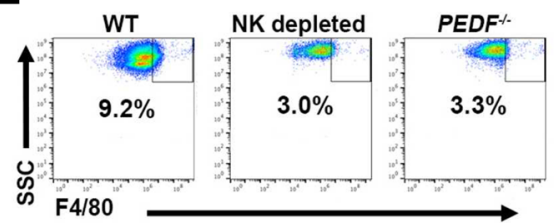

B

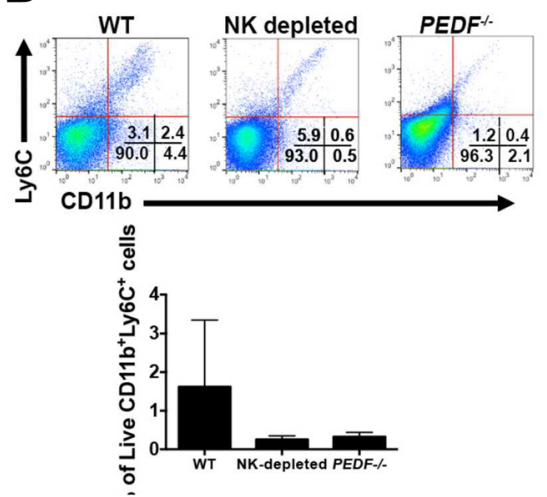

D
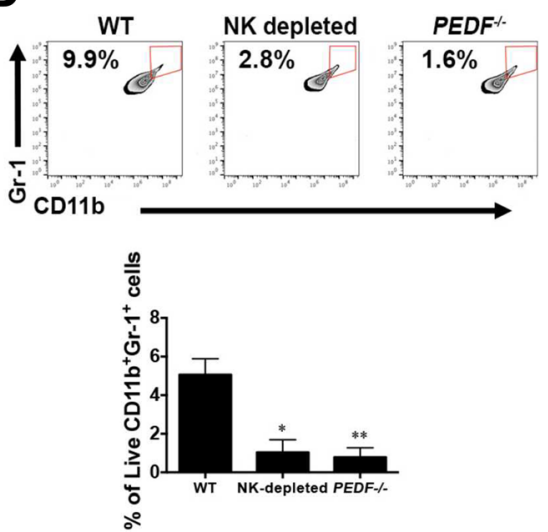

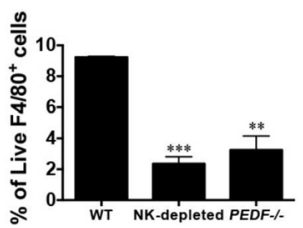

Fig. 4 Percentages of live CD11 $\mathrm{b}^{+}$, which comprise monocytes, neutrophils, dendritic cells, and some NK cells. a-e Another part of the livers was macerated followed by removal of red blood cells. Remaining cells were labeled with anti-CD $11 b^{+}$followed by flow cytometry analyses. a upper panel, Red gate indicates the percentage of $\mathrm{CD}_{1} \mathrm{~b}^{+}$cells. Lower panel shows bar graph summarizing the data. $\mathbf{b}$-e The CD11 $\mathrm{b}^{+}$analyses were subdivided based on the presence of cell surface markers to determine the percentage of CD11 b ${ }^{+} L y 6 C^{+}(\mathbf{b}), C_{1} 11 b^{+} L y 6 G^{+}(\mathbf{c}), C D_{11} b^{+} G r-1^{+}(\mathbf{d})$, and F4/80+ (e) cells. In Fig. 4a-c, and $\mathbf{d}$, the upper panel shows a representative pseudocolor FACS plot from each of the groups and the lower panel shows a bar graph summarizing the data. Figure 3d, upper panel shows a Zebra plot for each of the groups; lower panel shows bar graph with data summary. All data is represented as mean \pm SEM using The Holm-Sidak t-test; ${ }^{*} p<0.05,{ }^{*} p<0.005,{ }^{* *} p<0.0005$

followed stage $1>$ stage $2>$ stage 3 . Collectively, our work supports a critical role for NK cells in the control of hepatic metastases.

Multiple groups, including ours, have investigated M1/ M2 polarization as predictors of outcome in UM patients [26-33]. A number of molecular markers and cytokines are used to classify macrophages into M1 and M2. Classically, M1 are considered the destroyers of tumor cells. These M1 macrophages present antigen to $\mathrm{T}$ cells, produce high levels of pro-inflammatory cytokines, and express both nitric oxide synthase and reactive oxygen species $[34,35]$. In contrast, M2 suppress 
type-1 immune responses, promote tissue remodeling and wound healing, angiogenesis via VEGF and promote tumor development $[34,36]$. We examined the genetic expression of two markers associated with M1 and M2 polarization. The absence of NK cells measured similar expression of Socs3 mRNA to WT groups, associated with tumor killing, but base levels of $\operatorname{Tnf}$ mRNA, a key pro-inflammatory cytokine in anti-tumor responses. Similar Arg1 and Tgfb mRNA expression were observed in the absence of NK cells compared to WT groups. This raises multiple questions for further investigations on the plasticity of these macrophages and their contribution in angiogenesis and disease progression [37].

Tumor cell survival is associated with a decreased capacity of tumor suppressors, such as p53, to execute their function. P53-dependent apoptosis is induced by activation of effector caspases, such as caspase-3 [38]. In our investigation we measured reduction of both Casp3 and Trp53 mRNA in NK-depleted animals compared to WT. This data confirms caspase- 3 is an important effector molecule in NK cell-mediated apoptosis in tumors. The $P E F^{-1-}$ animals measured a stronger effect on downregulation of both Casp3 and Trp53 mRNA. Work from Takenaka and colleagues [39] recently demonstrated activation of caspase-3 and induction of apoptosis by PEDF using the MG63 human osteosarcoma cells. These results suggest PEDF, in addition to decreasing hepatic metastases by eliciting anti-angiogenic responses $[15,19]$ also regulates caspase- 3 activation. Based on the work performed in the human livers from metastatic UM patients [9] we hypothesized that the nodular pattern is controlled by PEDF in the periportal area, thus, absence of PEDF will increase the ratio of nodular to infiltrative growth. Our results supported our hypothesis as the $P E D F^{-1-}$ group had a significant increase in the metastatic growth following the nodular pattern compared to WT and NK depleted groups. Nodular metastases in human livers express MMP-9, VEGF, and contain CD $31^{+}$ vascular channels [9]. Therefore, we can speculate PEDF from hepatocytes inhibit the VEGF from the tumor microenvironment. Here, we provide evidence of the anti-angiogenic role of PEDF in the hepatic metastases as the $P E D F^{-1-}$ grafts showed a significant increase in MVD compared to WT.

Characterization of the myeloid-derived cells in the liver microenvironment suggests that dysfunction of multiple cells types can lead to failure to control hepatic metastases. Our results showed a reduction in

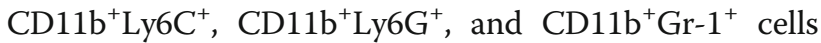
in the NK-depleted and $P E D F^{-/}$groups. Both groups failed to control the growth of hepatic metastases in our orthotopic ocular melanoma model. These results are consistent with the concept that the absence of tissue infiltrating myeloid-derived cells modifies the tumor microenvironment, leading to failure to control tumorigenesis [40, 41]. Neutrophils are considered of great importance in the control or the progression of different cancers [42-44]. However, the dual roles of neutrophils, as an anti-tumor and pro-tumor functions, needs to be further studied [45-49]. Recent work from Sadegh et al. [50], demonstrated the development of hepatic metastases to be associated with the upregulation of IL-10 in the liver microenvironment and its receptor on NK cells. The source of IL-10 was confined to bone marrow-derived cells that are not MDSCs. Sadegh et al., used Gr-1-depletion to investigate if MDSCs were the source of IL-10. It has been demonstrated $\mathrm{Gr}-1^{+}$is also a neutrophil marker; therefore, in our investigations we characterized cells based on $\mathrm{Gr}-1$ - and Ly6C/G-positivity. MDSCs have been considered pro-tumoral at the systemic level. Our work suggests that the presence of these cells have an anti-tumoral effect, as their percentages are reduced in those groups with elevated number of metastases. It is tempting to speculate their effector function is locally within the liver microenvironment.

In this investigation we demonstrated the two distinctive metastatic growth patterns in hepatic metastases of an orthotopic murine model of ocular melanoma. We demonstrated NK cells play a critical role in the control of hepatic metastases following an infiltrative growth pattern. In addition, we demonstrated the importance of PEDF in the control of angiogenesis in this animal model, which also contributes to the generation of large nodular growth. Together, our work provides a novel understanding of the infiltrative and nodular patters of metastases and on the role of myeloid-derived suppressor cells in metastatic UM.

\section{Conclusions}

The majority of cancer mortality is associated with tumor dissemination rather than the primary tumor. The development of metastases is correlated with poor prognosis and there are limitations for effective anti-metastatic treatments. Herein, we provide a mechanistic understanding of the growth patterns associated with hepatic metastases, namely infiltrative and nodular, using an orthotopic murine model of ocular melanoma. Furthermore, we characterized the myeloid immune compartment in the tumor microenvironment by flow cytometry analyses. These analyses revealed a reduction in macrophages and myeloid-derived suppressor cells (MDSCs), which promoted an anti-inflammatory response in both NK cell deficient and $P E D F^{-/-}$groups, suggesting MDSC reduction does not confer a survival advantage. Our findings represent a novel understanding of the infiltrative and nodular patters of metastases and on the role of MDSCs in metastatic UM. 


\section{Abbreviations}

APC: Allophycocyanin; Arg1: Arginase 1 gene; Casp3: Caspase-3 gene; CD31: Cluster of differentiation 31; CD68: Cluster of differentiation 68 gene; CDNA: Complementary deoxyribonucleic acid; c-Met: Tyrosine-protein kinase Met; CXCR4: C-X-C chemokine receptor type 4; FBS: Fetal bovine serum; FITC: Fluorescein isothyocyanate; FSC: Forward scatter; H\&E: Hematoxylin and eosin; HPF: High-powered field; i.p.: Intra-peritoneal; M1: Macrophage type-1; M2: Macrophage type-2; MDSC: Myeloid-derived suppressor cells; MEM: Minimum Essential Media; MIF: Macrophage migration inhibitory factor; mRNA: Messenger RNA; MVD: Mean vascular density; NK: Natural killer cells; P53: Tumor protein 53; PE: Phycoerythrin; PEDF: Pigment epithelial-derived factor; qPCR: Quantitative polymerase chain reaction; RNA: Ribonucleic aci; SEM: Standard error measurement; Socs3: Suppressor of cytokine signaling 3 gene; SSC: Side scatter; Tgfb: Transforming growth factor beta gene; TLR5: Toll-like receptor 5; Tnf: Tumor necrosis factor alpha gene; Trp53: Transformation related protein 53 gene; UM: Uveal melanoma; VEGF: Vascular endothelial growth factor; WT: Wild type

\section{Acknowledgements}

We would like to thank the members of the Grossniklaus laboratory for helpful discussions, Mr. Micah Chrenek and Ms. Jana Sellers at Emory University, Mr. Bradley T. Gao and Mr. Zachary K. Goldsmith at the University of Tennessee Health Science Center (UTHSC) for technical assistance.

\section{Funding}

This work was funded by the Gerwin Fellowship (VMT), 930 Friends (VMT), Research to Prevent Blindness (HEG), NIH P30EY06360 and NIH NCI R01CA176001 (HEG).

The funding body/bodies had no role in the design of the study and collection, analysis, and interpretation of data and in writing this manuscript.

\section{Availability of data and materials}

Anti-asialo GM1 (Asialo GM1 ganglioside) chemical structure data is available in PubChem Substance (pubchem.ncbi.nlm.nih.gov); PubChem CID: 6450363.

\section{Authors' contributions}

The conception and design of the work was done by HEG, HY, and VMT; data acquisition, analysis, and interpretation by NMJ, HY, QZ, VMT, and HEG; manuscript draft and significant contribution in intellectual content by $\mathrm{NMJ}$, HY QZ, VMT, and HEG. All authors approved final version of manuscript. The following authors have no competing interests: NMJ, HY, QZ, VMT, and HEG.

\section{Authors' information}

$\mathrm{HY}$ is an Associate Professor at the Research Section of the Department of Ophthalmology at Emory University, who focuses on anti-angiogenic and immune modulatory therapies against metastatic uveal melanoma. VMT is an Assistant Professor at the Departments of Ophthalmology and Microbiology, Immunology and Biochemistry (MIB) at the University of Tennessee Health Science Center. She leads the Ocular Oncology and Immunology Translational Research Unit at UTHSC and has expertise in human tumor immunology, intraocular tumors and pre-clinical models of disease. HEG serves as the Interim Vice Chair at the Emory Eye Center, founding director of the Ocular Oncology and Pathology Service and Director of the L.F. Montgomery Laboratory at Emory. HEG is considered an international expert in diagnostic ophthalmic pathology, and ocular oncology with over 500 publications in peer reviewed journals, over 25 book chapters and edited 10 books.

\section{Ethics approval and consent to participate}

This study was approved by the Emory University School of Medicine Institutional Animal Care and Utilization Committee (IACUC).

\section{Consent for publication}

Not applicable

\section{Competing interests}

The authors declare that they have no competing interests.

\section{Publisher's Note}

Springer Nature remains neutral with regard to jurisdictional claims in published maps and institutional affiliations.

\section{Author details}

Graduate Division of Biological and Biomedical Sciences Cancer Biology and Translational Oncology, Emory University, Atlanta, GA 30322, USA. ${ }^{2}$ Department of Ophthalmology, Emory University School of Medicine, Atlanta, GA 30322, USA. ${ }^{3}$ Department of Ophthalmology, Hamilton Eye Institute, University of Tennessee Health Science Center, Memphis, TN 38163, USA. ${ }^{4}$ Department of Microbiology, Immunology, and Biochemistry, University of Tennessee Health Science Center, Memphis, TN 38163, USA. ${ }^{5}$ Winship Cancer Institute at Emory University, 1365 Clifton Road NE, BT428, Atlanta, GA 30322, USA.

Received: 14 February 2018 Accepted: 14 May 2019

Published online: 22 May 2019

\section{References}

1. Woll E, Bedikian A, Legha SS. Uveal melanoma: natural history and treatment options for metastatic disease. Melanoma Res. 1999;9(6):575-81.

2. Miyamoto C, Balazsi M, Bakalian S, Fernandes BF, Burnier MN Jr. Uveal melanoma: ocular and systemic disease. Saudi J Ophthalmol. 2012;26(2):145-9.

3. Eagle RC Jr. The pathology of ocular cancer. Eye. 2013;27(2):128-36.

4. Li H, Yang W, Chen PW, Alizadeh H, Niederkorn JY. Inhibition of chemokine receptor expression on uveal melanomas by CXCR4 siRNA and its effect on uveal melanoma liver metastases. Invest Ophthalmol Vis Sci. 2009;50(12): $5522-8$.

5. Bi J, Li P, Li C, He J, Wang Y, Zhang H, Fan X, Jia R, Ge S. The SDF-1/CXCR4 chemokine axis in uveal melanoma cell proliferation and migration. Tumour Biol. 2016;37(3):4175-82.

6. Li H, Alizadeh H, Niederkorn JY. Differential expression of chemokine receptors on uveal melanoma cells and their metastases. Invest Ophthalmol Vis Sci. 2008:49(2):636-43.

7. Ye M, Hu D, Tu L, Zhou X, Lu F, Wen B, Wu W, Lin Y, Zhou Z, Qu J. Involvement of PI3K/Akt signaling pathway in hepatocyte growth factorinduced migration of uveal melanoma cells. Invest Ophthalmol Vis Sci. 2008; 49(2):497-504.

8. Hendrix MJ, Seftor EA, Seftor RE, Kirschmann DA, Gardner LM, Boldt HC, Meyer M, Pe'er J, Folberg R. Regulation of uveal melanoma interconverted phenotype by hepatocyte growth factor/scatter factor (HGF/SF). Am J Pathol. 1998;152(4):855-63.

9. Grossniklaus HE, Zhang Q, You S, McCarthy C, Heegaard S, Coupland SE. Metastatic ocular melanoma to the liver exhibits infiltrative and nodular growth patterns. Hum Pathol. 2016;57:165-75.

10. Van den Eynden GG, Majeed AW, Illemann M, Vermeulen PB, Bird NC, Hoyer-Hansen G, Eefsen RL, Reynolds AR, Brodt P. The multifaceted role of the microenvironment in liver metastasis: biology and clinical implications. Cancer Res. 2013;73(7):2031-43.

11. Apte RS, Mayhew E, Niederkorn JY. Local inhibition of natural killer cell activity promotes the progressive growth of intraocular tumors. Invest Ophthalmol Vis Sci. 1997;38(6):1277-82.

12. Repp AC, Mayhew ES, Apte S, Niederkorn JY. Human uveal melanoma cells produce macrophage migration-inhibitory factor to prevent lysis by NK cells. J Immunol. 2000;165(2):710-5.

13. Yang H, Brackett CM, Morales-Tirado VM, Li Z, Zhang Q, Wilson MW, Benjamin C, Harris W, Waller EK, Gudkov AV, et al. The toll-like receptor 5 agonist entolimod suppresses hepatic metastases in a murine model of ocular melanoma via an NK cell-dependent mechanism. Oncotarget. 2016; 7(3):2936-50.

14. Yang $\mathrm{H}, \mathrm{Xu} Z$ Z, luvone PM, Grossniklaus HE. Angiostatin decreases cell migration and vascular endothelium growth factor (VEGF) to pigment epithelium derived factor (PEDF) RNA ratio in vitro and in a murine ocular melanoma model. Mol Vis. 2006;12:511-7.

15. Yang $\mathrm{H}$, Grossniklaus HE. Constitutive overexpression of pigment epithelium-derived factor inhibition of ocular melanoma growth and metastasis. Invest Ophthalmol Vis Sci. 2010;51(1):28-34.

16. Doll JA, Stellmach VM, Bouck NP, Bergh AR, Lee C, Abramson LP, Cornwell ML, Pins MR, Borensztajn J, Crawford SE. Pigment epithelium-derived factor regulates the vasculature and mass of the prostate and pancreas. Nat Med. 2003;9(6):774-80.

17. Yang $\mathrm{H}$, Dithmar $\mathrm{S}$, Grossniklaus HE. Interferon alpha $2 \mathrm{~b}$ decreases hepatic micrometastasis in a murine model of ocular melanoma by activation of intrinsic hepatic natural killer cells. Invest Ophthalmol Vis Sci. 2004:45(7): 2056-64. 
18. Diaz CE, Rusciano D, Dithmar S, Grossniklaus HE. B16LS9 melanoma cells spread to the liver from the murine ocular posterior compartment (PC). Curr Eye Res. 1999;18(2):125-9.

19. Lattier JM, Yang H, Crawford S, Grossniklaus HE. Host pigment epitheliumderived factor (PEDF) prevents progression of liver metastasis in a mouse model of uveal melanoma. Clin Exp Metastasis. 2013;30(8):969-76.

20. Gao BT, Lee RP, Jiang Y, Steinle JJ, Morales-Tirado VM. Pioglitazone alters monocyte populations and stimulates recent thymic emigrants in the BBDZR/Wor type 2 diabetes rat model. Diabetol Metab Syndr. 2015;7:72.

21. Webb AH, Gao BT, Goldsmith ZK, Irvine AS, Saleh N, Lee RP, Lendermon JB, Bheemreddy R, Zhang Q, Brennan RC, et al. Inhibition of MMP-2 and MMP-9 decreases cellular migration, and angiogenesis in in vitro models of retinoblastoma. BMC Cancer. 2017;17(1):434.

22. Nielsen HV, Christensen JP, Andersson EC, Marker O, Thomsen AR. Expression of type 3 complement receptor on activated CD8+ T cells facilitates homing to inflammatory sites. J Immunol. 1994;153(5):2021-8.

23. Springer T, Galfre G, Secher DS, Milstein C. Mac-1: a macrophage differentiation antigen identified by monoclonal antibody. Eur J Immunol. 1979;9(4):301-6.

24. Kay HD, Horwitz DA. Evidence by reactivity with hybridoma antibodies for a probable myeloid origin of peripheral blood cells active in natural cytotoxicity and antibody-dependent cell-mediated cytotoxicity. J Clin Invest. 1980;66(4):847-51.

25. Zarling JM, Kung PC. Monoclonal antibodies which distinguish between human NK cells and cytotoxic T lymphocytes. Nature. 1980;288(5789):394-6.

26. Herwig MC, Bergstrom C, Wells JR, Holler T, Grossniklaus HE. M2/M1 ratio of tumor associated macrophages and PPAR-gamma expression in uveal melanomas with class 1 and class 2 molecular profiles. Exp Eye Res. 2013;107:52-8.

27. Herwig MC, Grossniklaus HE. Role of macrophages in uveal melanoma. Expert Rev Ophthalmol. 2011;6(4):405-7.

28. Jager MJ, Ly LV, El Filali M, Madigan MC. Macrophages in uveal melanoma and in experimental ocular tumor models: friends or foes? Prog Retin Eye Res. 2011;30(2):129-46.

29. Bronkhorst $I H$, Ly LV, Jordanova ES, Vrolijk J, Versluis M, Luyten GP, Jager MJ. Detection of M2-macrophages in uveal melanoma and relation with survival. Invest Ophthalmol Vis Sci. 2011;52(2):643-50.

30. Ly LV, Baghat A, Versluis M, Jordanova ES, Luyten GP, van Rooijen N, van Hall T, van der Velden PA, Jager MJ. In aged mice, outgrowth of intraocular melanoma depends on proangiogenic M2-type macrophages. J Immunol. 2010;185(6):3481-8.

31. Makitie T, Summanen P, Tarkkanen A, Kivela T. Tumor-infiltrating macrophages (CD68(+) cells) and prognosis in malignant uveal melanoma. Invest Ophthalmol Vis Sci. 2001;42(7):1414-21.

32. Torisu H, Ono M, Kiryu H, Furue M, Ohmoto Y, Nakayama J, Nishioka Y, Sone S, Kuwano M. Macrophage infiltration correlates with tumor stage and angiogenesis in human malignant melanoma: possible involvement of TNFalpha and IL-1alpha. Int J Cancer. 2000;85(2):182-8.

33. COMS. Histopathologic characteristics of uveal melanomas in eyes enucleated from the collaborative ocular melanoma study. COMS report no. 6. Am J Ophthalmol. 1998;125(6):745-66

34. Martinez FO, Gordon S. The M1 and M2 paradigm of macrophage activation: time for reassessment. F1000prime Rep. 2014;6:13.

35. Italiani $\mathrm{P}$, Boraschi D. From monocytes to M1/M2 macrophages: phenotypical vs. functional differentiation. Front Immunol. 2014;5:514

36. Tham M, Tan KW, Keeble J, Wang X, Hubert S, Barron L, Tan NS, Kato M, Prevost-Blondel A, Angeli $\mathrm{V}$, et al. Melanoma-initiating cells exploit M2 macrophage TGFbeta and arginase pathway for survival and proliferation. Oncotarget. 2014;5(23):12027-42.

37. Gezgin G, Dogrusoz M, van Essen TH, Kroes WGM, Luyten GPM, van der Velden PA, Walter V, Verdijk RM, van Hall T, van der Burg SH, et al. Genetic evolution of uveal melanoma guides the development of an inflammatory microenvironment. Cancer Immunol Immunother. 2017;66(7):903-12.

38. Schuler M, Bossy-Wetzel E, Goldstein JC, Fitzgerald P, Green DR. p53 induces apoptosis by caspase activation through mitochondrial cytochrome c release. J Biol Chem. 2000;275(10):7337-42.

39. Takenaka K, Yamagishi S, Jinnouchi Y, Nakamura K, Matsui T, Imaizumi T. Pigment epithelium-derived factor (PEDF)-induced apoptosis and inhibition of vascular endothelial growth factor (VEGF) expression in MG63 human osteosarcoma cells. Life Sci. 2005;77(25):3231-41.
40. Nagaraj S, Collazo M, Corzo CA, Youn II, Ortiz M, Quiceno D, Gabrilovich DI. Regulatory myeloid suppressor cells in health and disease. Cancer Res. 2009; 69(19):7503-6.

41. Gabrilovich DI, Nagaraj S. Myeloid-derived suppressor cells as regulators of the immune system. Nat Rev Immunol. 2009;9(3):162-74.

42. Gregory AD, Houghton AM. Tumor-associated neutrophils: new targets for cancer therapy. Cancer Res. 2011;71(7):2411-6.

43. Brandau S, Trellakis S, Bruderek K, Schmaltz D, Steller G, Elian M, Suttmann H, Schenck M, Welling J, Zabel P, et al. Myeloid-derived suppressor cells in the peripheral blood of cancer patients contain a subset of immature neutrophils with impaired migratory properties. J Leukoc Biol. 2011;89(2):311-7.

44. Schmidt H, Suciu S, Punt CJ, Gore M, Kruit W, Patel P, Lienard D, von der Maase $H$, Eggermont AM, Keilholz U, et al. Pretreatment levels of peripheral neutrophils and leukocytes as independent predictors of overall survival in patients with American joint committee on Cancer stage IV melanoma: results of the EORTC 18951 biochemotherapy trial. J Clin Oncol. 2007;25(12): $1562-9$

45. Hayata K, Iwahashi M, Ojima T, Katsuda M, lida T, Nakamori M, Ueda K, Nakamura M, Miyazawa M, Tsuji T, et al. Inhibition of IL-17A in tumor microenvironment augments cytotoxicity of tumor-infiltrating lymphocytes in tumor-bearing mice. PLoS One. 2013:8(1):e53131.

46. Di Carlo E, Forni G, Lollini P, Colombo MP, Modesti A, Musiani P. The intriguing role of polymorphonuclear neutrophils in antitumor reactions. Blood. 2001;97(2):339-45.

47. Mishalian I, Bayuh R, Levy L, Zolotarov L, Michaeli J, Fridlender ZG. Tumorassociated neutrophils (TAN) develop pro-tumorigenic properties during tumor progression. Cancer Immunol Immunother. 2013;62(11):1745-56.

48. Fridlender ZG, Sun J, Mishalian I, Singhal S, Cheng G, Kapoor V, Horng W, Fridlender G, Bayuh R, Worthen GS, et al. Transcriptomic analysis comparing tumor-associated neutrophils with granulocytic myeloid-derived suppressor cells and normal neutrophils. PLoS One. 2012;7(2):e31524.

49. Piccard H, Muschel RJ, Opdenakker G. On the dual roles and polarized phenotypes of neutrophils in tumor development and progression. Crit Rev Oncol Hematol. 2012:82(3):296-309.

50. Sadegh L, Chen PW, Brown JR, Han Z, Niederkorn JY. NKT cells act through third party bone marrow-derived cells to suppress NK cell activity in the liver and exacerbate hepatic melanoma metastases. Int J Cancer. 2015; 137(5):1085-94.
Ready to submit your research? Choose BMC and benefit from:

- fast, convenient online submission

- thorough peer review by experienced researchers in your field

- rapid publication on acceptance

- support for research data, including large and complex data types

- gold Open Access which fosters wider collaboration and increased citations

- maximum visibility for your research: over $100 \mathrm{M}$ website views per year

At $\mathrm{BMC}$, research is always in progress.

Learn more biomedcentral.com/submissions 